\title{
BMJ Open Are self-reported telemonitored blood pressure readings affected by end-digit preference: a prospective cohort study in Scotland
}

Richard A Parker, ${ }^{1}$ Mary Paterson, ${ }^{1}$ Paul Padfield, ${ }^{1}$ Hilary Pinnock, ${ }^{1}$ Janet Hanley, ${ }^{2}$ Vicky S Hammersley, ${ }^{1}$ Adam Steventon, ${ }^{3}$ Brian McKinstry ${ }^{1}$

To cite: Parker RA, Paterson M, Padfield $P$, et al. Are selfreported telemonitored blood pressure readings affected by end-digit preference: a prospective cohort study in Scotland. BMJ Open 2018;8:e019431. doi:10.1136/ bmjopen-2017-019431

\section{- Prepublication history and} additional material for this paper are available online. To view these files, please visit the journal online (http://dx.doi. org/10.1136/bmjopen-2017019431).

Received 31 August 2017 Revised 25 0ctober 2017 Accepted 7 December 2017

\section{Check for updates}

${ }^{1}$ Usher Institute of Population Health Sciences and Informatics, University of Edinburgh, Edinburgh, UK

${ }^{2}$ School of Health and Social Care, Edinburgh Napier University, Edinburgh, UK

${ }^{3}$ The Health Foundation, London, UK

Correspondence to

Richard A Parker;

richard.parker@ed.ac.uk

\section{ABSTRACT}

Objective Simple forms of blood pressure (BP)

telemonitoring require patients to text readings to central servers creating an opportunity for both entry error and manipulation. We wished to determine if there was an apparent preference for particular end digits and entries which were just below target BPs which might suggest evidence of data manipulation.

Design Prospective cohort study

Setting 37 socioeconomically diverse primary care practices from South East Scotland.

Participants Patients were recruited with hypertension to a telemonitoring service in which patients submitted home $\mathrm{BP}$ readings by manually transcribing the measurements into text messages for transmission ('patient-texted system'). These readings were compared with those from primary care patients with uncontrolled hypertension using a system in which readings were automatically transmitted, eliminating the possibility of manipulation of values ('automatic-transmission system').

Methods A generalised estimating equations method was used to compare BP readings between the patient-texted and automatic-transmission systems, while taking into account clustering of readings within patients.

Results A total of $44150 \mathrm{BP}$ readings were analysed on 1068 patients using the patient-texted system compared with 20705 readings on 199 patients using the automatic-transmission system. Compared with the automatic-transmission data, the patient-texted data showed a significantly higher proportion of occurrences of both systolic and diastolic BP having a zero end digit (OR $2.1,95 \% \mathrm{Cl} 1.7$ to 2.6 ) although incidence was $<2 \%$ of readings. Similarly, there was a preference for systolic 134 and diastolic 84 (the threshold for alerts was 135/85) (134 systolic BP OR 1.5, 95\% Cl 1.3 to 1.8; 84 diastolic BP OR $1.5,95 \% \mathrm{Cl} 1.3$ to 1.9 ).

Conclusion End-digit preference for zero numbers and specific-value preference for readings just below the alert threshold exist among patients in self-reporting their BP using telemonitoring. However, the proportion of readings affected is small and unlikely to be clinically important.

Trial registration number ISRCTN72614272; Postresults.
Strengths and limitations of this study

- This study involved the analysis of a very large sample size of over 44000 patient-texted blood pressure (BP) readings recorded by 1068 patients.

- We compared with a cohort of 20705 automatically transmitted telemonitored BP readings recorded by 199 patients for which there was no possibility of manipulation of readings.

- Because of differential recruitment criteria, the 'automatic-transmission' cohort had higher BP readings than the 'patient-texted' cohort although this was addressed to some extent in the statistical analysis.

\section{INTRODUCTION}

Hypertension is one of the most important risk factors for stroke and heart disease, and is estimated to contribute to 7.6 million premature deaths each year worldwide. ${ }^{1}$ Although antihypertensive medication is highly effective, control of blood pressure (BP) is often poor in routine clinical practice. ${ }^{2}$ Reasons for poor BP control include irregular monitoring, ${ }^{3}$ reluctance of physicians to intensify medications ${ }^{4}$ and poor adherence to medication by patients. ${ }^{5}$

$\mathrm{BP}$ varies considerably over time, so infrequent measurements as part of routine clinical reviews are often poor indicators of response to therapy. ${ }^{6}$ Furthermore, clinic BP measurements have poor test-retest reliability compared with daytime ambulatory BP monitoring or multiple measurements taken at home, ${ }^{7}$ and are less predictive of mortality or cardiovascular outcomes. ${ }^{8-10}$

Telemonitoring of hypertension involves patients submitting home BP readings directly to their clinician using the internet or other technology, with the data being used to assist with diagnosis or ongoing management. A recent meta-analysis of randomised 
controlled trials concluded that telemonitoring results in a clinically significant reduction in systolic and diastolic BP. ${ }^{11}$ This has also been shown more generally with respect to self-monitoring of $\mathrm{BP}$ in hypertensive patients, when accompanied with co-interventions involving additional support, which is based on the results from an individual patient data meta-analysis. ${ }^{12}$

A low-cost telemonitoring BP service is currently being rolled out in primary care in NHS Lothian in Scotland. The telemonitoring platform used in this study involves patients checking their BP at home with a frequency determined by their general practitioner according to the clinical context. For example, some patients took daily readings, some twice weekly and others monthly. The patients had to transcribe the readings into text messages to send to the system which generated summary reports for their practice at one to three monthly intervals. If readings were above clinically determined BP thresholds, then this would trigger an alert from the system. Different thresholds and recommended actions were used depending on the patient's condition as per guidelines in place at the time. For people with diabetes or chronic kidney disease (CKD), the threshold for alerts was typically set at 125/75, whereas for most other patients it was 135/85.

The manual transcribing of measurements required by this system contrasts with a system previously used in a trial in Lothian in which Bluetooth-connected sphygmomanometers automatically transmitted data so that there was no possibility of transcribing errors or value manipulation..$^{13}$ There were concerns with the new patient-texted system that patients might provide inaccurate readings.

Many studies have shown a tendency for nurses and physicians throughout the world to record BP readings to the nearest zero ('end-digit preference'). ${ }^{14-25}$ Indeed, in a recent meta-analysis published in $2017,{ }^{26}$ as many as 34 studies were identified that reported over-representation of zero end digits in BP readings by as much as $71 \%{ }^{20}{ }^{26}$ In part, this is due to historical sphygmomanometers requiring manual estimation of $\mathrm{BP}$, and the phenomenon is reduced (but not eliminated) with automatic digital monitors. ${ }^{14}$ In addition, there is a tendency for readings taken by professionals to cluster just below treatment threshold values. This has been investigated in a few studies, ${ }^{14} 172425$ but results are inconsistent, and the clinical impact is unclear.

To our knowledge, no studies to date have examined end-digit preference of BP readings measured and texted by patients. The SMART study considered end-digit preference in self-reported BP measurements recorded by patients at home, although this was not in the context of a telemonitoring system. ${ }^{27}$ Steventon $e t a l^{28}$ found evidence of end-digit preference in weight measures among patients with heart failure using telemonitoring. However, neither study had a comparator group using automatically transmitted data in which there was no possibility of data manipulation.

Our hypothesis was that if a proportion of patients are estimating or manipulating the BP values that they submit to the system, then there would also be a preference for zero end digits. We were also interested in whether there was a specific-value preference where patients were preferentially recording BP readings just below the BP thresholds in order to avoid triggering an alert.

We therefore aimed to investigate the occurrence of end-digit bias and specific-value preference in BP readings taken using the patient-texted telemonitoring system by comparison with readings from the automatic-transmission system.

\section{METHODS AND ANALYSIS}

There were two cohorts of patients included in the study: one from a telemonitoring service rollout involving a patient-texted telemonitoring system and one from a, previously published, randomised controlled trial using an automated telemonitoring system. ${ }^{13}$ Further details of each of these cohorts are given below.

\section{Scale-up BP: a system using 'patient-texted' BP readings}

Thirty-seven family practices volunteered to use a textbased telemonitoring system as an option for routine care in the 'Scale-up BP' initiative in NHS Lothian, Scotland. Data were collected from all patients recruited to the telemonitoring system from the 37 practices between 2 September 2015 and 30 June 2017.

Health professionals within each practice identified patients with hypertension who normally attended the primary care practice for $\mathrm{BP}$ monitoring. Patients were eligible to use the text-based telemonitoring system (Florence, https:/ /www.getflorence.co.uk/) if they were named on the practice hypertension register, had newly diagnosed hypertension or their BP control needed careful management to optimise use of antihypertensive medication.

Eligible patients were either given a leaflet during a routine appointment or sent a letter from the practice nurse or doctor outlining the telemonitoring approach to managing BP. They were asked to bring their mobile phone (if they had one) to their next appointment. If the patient (or a member of their family) were able and willing to use the telemonitoring system, they were given an Omron sphygmomanometer ${ }^{29}$ with an appropriate sized cuff, and the text-based telemonitoring system was demonstrated. The system was set to deliver a text reminder to measure $\mathrm{BP}$ at a frequency determined by the patient's clinician according to the clinical context. After training, patients were asked to demonstrate their ability to take their BP and respond to the text prompts. The general practitioner (GP) or practice nurse reviewed a summary of BPs sent to the practice at least three monthly and contacted patients if there were any problems. The patients were informed that the system was not monitored on a daily basis, and very high BPs, changes in readings or any problems with their medication should be reported in the usual way. Patients undertaking telemonitoring were asked to consent to their data to be used for evaluation of the system. The Scale-up BP study was considered by the 
Table 1 Descriptive statistics for systolic and diastolic blood pressure (BP) in the patient-texted and automatic-transmission systems

\begin{tabular}{lllll}
\hline & $\begin{array}{l}\text { Patient-texted } \\
\text { systolic BP }\end{array}$ & $\begin{array}{l}\text { Automatic-transmission } \\
\text { systolic BP }\end{array}$ & $\begin{array}{l}\text { Patient-texted } \\
\text { diastolic BP }\end{array}$ & $\begin{array}{l}\text { Automatic-transmission } \\
\text { diastolic BP }\end{array}$ \\
\hline N & 44150 & 20705 & 44150 & 20705 \\
Mean & 131.87 & 141.74 & 79.73 & 85.36 \\
SD & 14.87 & 15.07 & 9.96 & 12.27 \\
Median & 131 & 141 & 80 & 85 \\
Mode & 134 & 144 & 80 & 86 \\
IQR & 123 to 140 & 131 to 150 & 73 to 85 & 78 to 93 \\
Min & 70 & 75 & 40 & 30 \\
Max & 225 & 224 & 124 & 135 \\
\hline
\end{tabular}

local ethics scientific officer to be service evaluation and thus not to require ethical approval.

Data were obtained in monthly spreadsheets from a secure online web page. The spreadsheets were stripped of patient identifiers and did not include any sociodemographic data which meant that it was impossible to identify individual patients. After receiving the data, the following criteria were applied to exclude any highly improbable $\mathrm{BP}$ readings or clear input errors:

- systolic value below $60 \mathrm{~mm} \mathrm{Hg}$ or above $262 \mathrm{~mm} \mathrm{Hg}$

- diastolic value below $40 \mathrm{~mm} \mathrm{Hg}$ or above $124 \mathrm{~mm} \mathrm{Hg}$

- systolic lower than diastolic value

- systolic value less than $10 \mathrm{~mm} \mathrm{Hg}$ higher than diastolic value

- missing systolic or diastolic values.

Health Impact of nurse-led Telemetry Services trial: a system using 'automatically-transmitted' BP readings

As a comparator, we used BP data collected during the Health Impact of nurse-led Telemetry Services (HITS) trial, ${ }^{13}$ a multicentre randomised controlled trial in 20 primary care practices in the same health board as for the Scale-up BP study. A total of 401 people aged 29-95 years with uncontrolled BP (mean daytime ambulatory $\mathrm{BP} \geq 135 / 85 \mathrm{~mm} \mathrm{Hg}$ but $\leq 210 / 135 \mathrm{~mm} \mathrm{Hg}$ ) were recruited. The trial methods and results are reported in detail elsewhere. ${ }^{13}$ The patients used a validated sphygmomanometer (Stabil-O-Graph $)^{30}$ linked by a short range wireless connection to a mobile phone, which automatically transmitted readings to a central server, such that it was not possible to manipulate the value of the $\mathrm{BP}$ readings.

For the HITS trial, ${ }^{13}$ patients were recruited between February 2009 and October 2010. Patients were eligible if their last recorded clinic BP was over $145 \mathrm{~mm} \mathrm{Hg}$ systolic or $85 \mathrm{~mm} \mathrm{Hg}$ diastolic and they were on their practice hypertension register. They subsequently underwent daytime ambulatory BP monitoring to exclude those with 'white coat' hypertension. Exclusion criteria were secondary hypertension, hypertension or renal disease managed in secondary care, atrial fibrillation, diabetes, pregnancy, stroke, treatment for cardiac event or other life-threatening illness within the past 6 months or surgery within the last 3 months, under 18 years and inability to use self-monitoring equipment. Patients received automatic feedback from the system informing them that their BP was controlled $(<135 / 85)$, not yet controlled but improving, or that it was uncontrolled and that they should contact their clinician. Only anonymised BP readings from the telemonitoring system arm of the trial were made available for this analysis. The target for the HITS trial was the same as Scale-Up BP (135/85) although there were no participants with lower targets. The practice nurse monitored the readings as in Scale-up BP.

Written informed consent was obtained from participants in both studies.

\section{Analysis methods}

Overall descriptive statistics were calculated for the patienttexted and automatic-transmission BP readings. An independent samples t-test was used to compare mean systolic and diastolic BP between cohorts after first aggregating the data to the patient level and calculating a mean value per patient. The prevalence of specific values of systolic and diastolic BP readings was compared between the two data transmission cohorts. We then examined the percentage occurrence of all possible end digits (from 0 to 9), comparing between cohorts using clustered bar charts for systolic and diastolic BP. A cross tabulation of the end digits for systolic and diastolic within each dataset allowed to us to examine for the possibility of 'double zero' end-digit preference, where 'double zero' means that both systolic and diastolic BP have a zero end digit.

We used generalised estimating equations (GEE) analysis to directly compare between patient-texted/automatic-transmission data while taking into account clustering of observations by patients. To assess zero end-digit preference, binomial GEE models were fitted to the outcomes: double zero end digits, zero systolic end digit and zero diastolic end digit.

To assess specific-value preference of values, we were interested in systolic and diastolic values just below the hypertension threshold in both studies for alerts of $135 / 85$. Therefore, we fitted binomial GEE models to the specific-value outcomes of systolic 134 and diastolic 
Data source

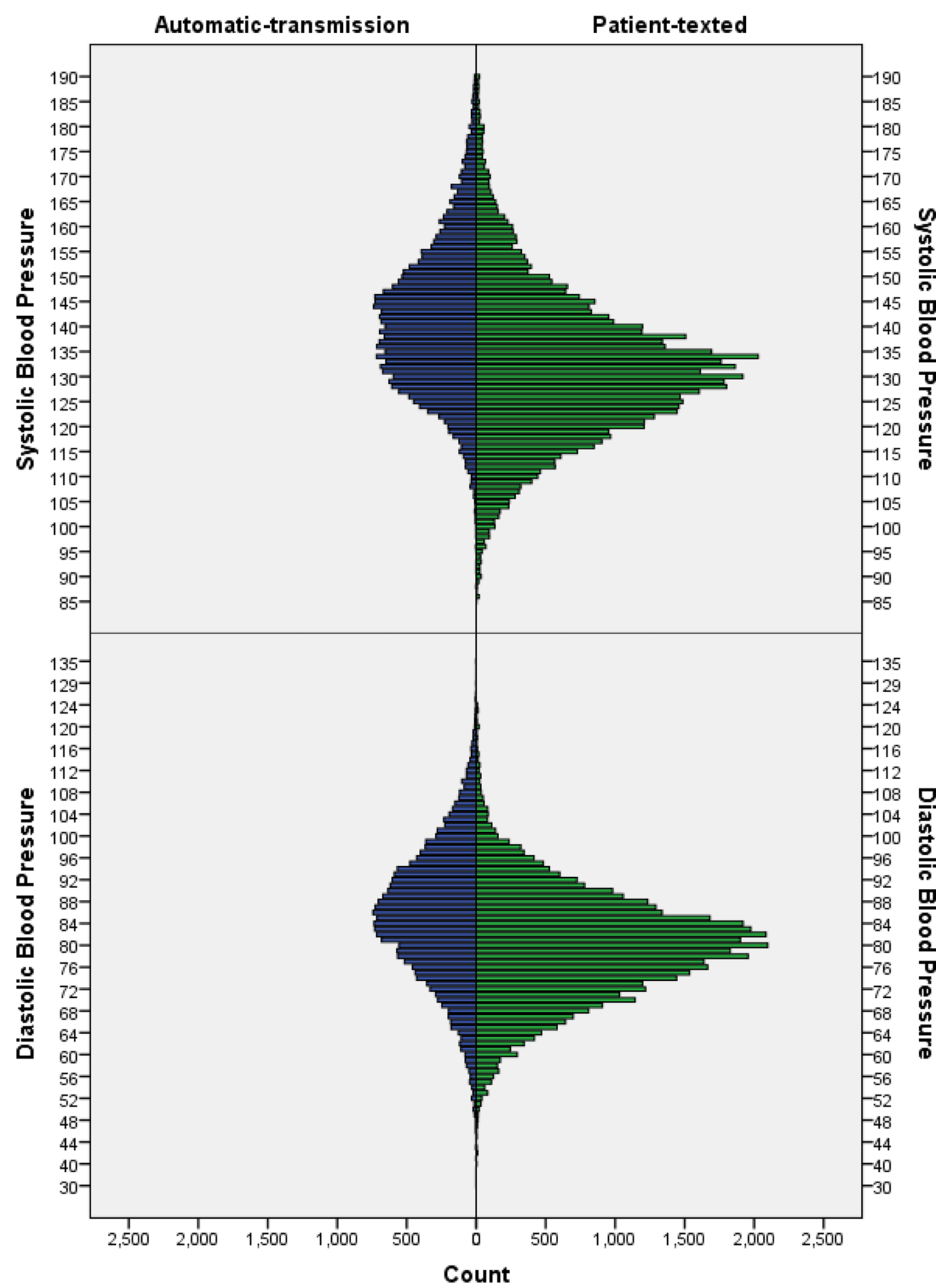

Figure 1 Population pyramids of the raw systolic blood pressure readings (in the range of 85-190) and diastolic readings (in the range of 30-135) in the patient-texted and automatic-transmission systems.

84. Unlike for other BP thresholds (eg, 120/80), these values are not confounded by double-zero preference. This analysis excluded patients from the patient-texted cohort using personalised limits other than the standard threshold for alerts (ie, 135/85). To address confounding due to population-level differences in the level of BP control, we also confined the samples in both groups to only include patients with median BPs in a restricted range (130-138 for systolic, and $82-86$ for diastolic). This was to make the patient samples as similar as possible in terms of level of BP control.

In sensitivity analysis, we performed the same analysis as above, but restricting the subgroup of patients to those submitting at least 50 readings. This allowed us to investigate if end-digit preference was present even among highly engaged individuals. 


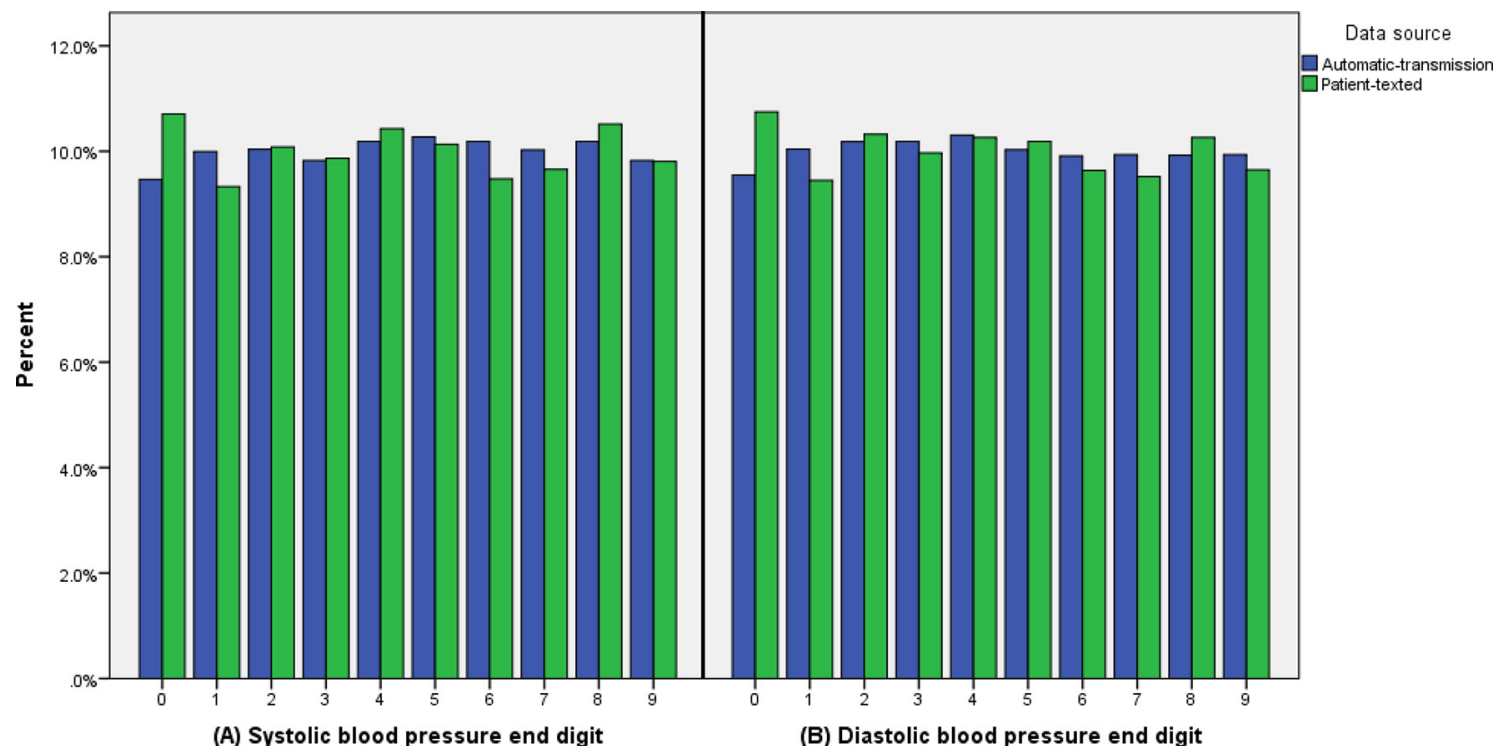

All GEE models assumed an exchangeable correlation structure. Analysis was by 'complete cases' only, and so incomplete BP readings were excluded.

The 'gee' package ${ }^{31}$ in $\mathrm{R}$ software ${ }^{32}$ was used for the GEE analysis. All other statistical analyses and graphs were produced using SPSS software V.21. ${ }^{33}$

\section{RESULTS}

A total of $44269 \mathrm{BP}$ readings were taken by 1069 patients using the patient-texted system. There were no missing systolic readings. Seventy-one readings were excluded because the diastolic value was missing. The following readings were excluded because they were implausible. Sixty-one systolic readings were below $60 \mathrm{~mm} \mathrm{Hg}, 17$ systolic readings were above $262 \mathrm{~mm} \mathrm{Hg}, 17$ diastolic readings were below $40 \mathrm{~mm} \mathrm{Hg}, 11$ diastolic readings were above $124 \mathrm{~mm} \mathrm{Hg}, 12$ readings had systolic BP lower than diastolic and a further 8 readings had systolic value within $10 \mathrm{~mm} \mathrm{Hg}$ of the diastolic value. After applying these exclusion criteria, we had a final cleaned dataset consisting of 44150 readings from 1068 patients.

The automatic-transmission data consisted of 20705 readings on 199 patients. There was a median of 18 readings per patient for the patient-texted data (IQR 8-41, range 1-786 readings), compared with a median of 80 readings per patient for the automatic-transmission data (IQR 59-124, range 1-559 readings). A total of 211 patients took over 50 readings in the patienttexted data (20\%) compared with 171 patients in the automatic transmission $(86 \%)$. Patients in the patienttexted group used the system for a median of 22 weeks (range 0-93 weeks) compared with a median of 26 weeks (range 0-42 weeks) for those in the automatic-transmission system group.

\section{Analysis of raw BP values}

Descriptive statistics for the patient-texted BP readings compared with the automatic-transmission readings are shown in table 1 .

Reflecting the different eligibility criteria, the patienttexted cohort had mean systolic and diastolic values that were significantly lower than in the automatic-transmission cohort (t-test $\mathrm{P}<0.001$ for both).

Population pyramids of the systolic and diastolic BP data are shown in figure 1.

In the patient-texted cohort, the highest percentage frequencies were observed for systolic BPs in the range of $127-135$ (2.9\% or above), and the most frequently observed was a value of systolic 134 (1623 occurrences, $3.7 \%$ ). Systolic 134 was also one of the most frequently observed systolic values in the automatic-transmission cohort, although the percentage occurrence was only $2.8 \%$.

For diastolic BPs in the patient-texted cohort, the highest percentage frequencies were observed for readings in the range of 78-84 (all above 4\%), with the most frequently observed diastolic value being $80(4.8 \%)$. In contrast, the percentage occurrence of 80 was only $2.7 \%$ in the automatic-transmission cohort, whereas the most frequent diastolic value had a percentage of $3.6 \%$ (86).

\section{Analysis of BP end digits}

Figure 2 shows clustered bar charts for the systolic and diastolic BP end digits, respectively.

The most notable difference between the two datasets regarding end-digit frequencies was for the zero end digit, indicating possible zero end-digit preference for both systolic and diastolic BP. In the patient-texted dataset, 4727 zero end digits were observed for systolic 
Table 2 Cross tabulation of systolic and diastolic end-digit readings for patient-texted and automatic-transmission datasets, with results shown as percentages of the total number of readings

\begin{tabular}{|c|c|c|c|c|c|c|c|c|c|c|c|c|c|}
\hline \multirow{2}{*}{ Data source } & & & \multicolumn{10}{|c|}{ Diastolic blood pressure end digit (\%) } & \multirow[b]{2}{*}{ Total (\%) } \\
\hline & & & 0 & 1 & 2 & 3 & 4 & 5 & 6 & 7 & 8 & 9 & \\
\hline \multirow{11}{*}{$\begin{array}{l}\text { Automatic- } \\
\text { transmission } \\
\text { system }\end{array}$} & \multirow{10}{*}{$\begin{array}{l}\text { Systolic blood } \\
\text { pressure end digit }\end{array}$} & 0 & 0.9 & 1.0 & 1.0 & 0.9 & 0.9 & 0.9 & 1.0 & 1.0 & 1.0 & 0.9 & 9.5 \\
\hline & & 1 & 1.0 & 1.0 & 0.9 & 1.0 & 1.0 & 1.0 & 1.0 & 0.9 & 1.0 & 1.0 & 10.0 \\
\hline & & 2 & 0.9 & 1.1 & 0.9 & 1.0 & 1.0 & 1.0 & 1.0 & 1.0 & 1.0 & 1.0 & 10.0 \\
\hline & & 3 & 1.0 & 1.0 & 1.0 & 1.0 & 1.0 & 1.0 & 0.9 & 1.0 & 1.0 & 0.9 & 9.8 \\
\hline & & 4 & 1.0 & 1.1 & 1.2 & 1.0 & 1.1 & 0.9 & 1.0 & 1.1 & 0.9 & 1.0 & 10.2 \\
\hline & & 5 & 1.0 & 1.0 & 1.0 & 1.0 & 1.2 & 1.0 & 1.0 & 1.1 & 0.9 & 1.0 & 10.3 \\
\hline & & 6 & 1.0 & 1.0 & 1.1 & 1.0 & 0.9 & 1.0 & 1.0 & 1.0 & 1.1 & 1.1 & 10.2 \\
\hline & & 7 & 1.0 & 1.0 & 1.1 & 1.0 & 1.1 & 1.0 & 1.0 & 1.0 & 1.0 & 0.9 & 10.0 \\
\hline & & 8 & 1.0 & 1.0 & 1.0 & 1.1 & 1.1 & 1.1 & 1.0 & 1.0 & 0.9 & 1.0 & 10.2 \\
\hline & & 9 & 0.9 & 1.0 & 1.0 & 1.1 & 1.0 & 1.1 & 0.9 & 0.8 & 0.9 & 1.1 & 9.8 \\
\hline & Total & & 9.5 & 10.0 & 10.2 & 10.2 & 10.3 & 10.0 & 9.9 & 9.9 & 9.9 & 9.9 & 100.0 \\
\hline \multirow{11}{*}{$\begin{array}{l}\text { Patient-texted } \\
\text { system }\end{array}$} & \multirow{10}{*}{$\begin{array}{l}\text { Systolic blood } \\
\text { pressure end digit }\end{array}$} & 0 & 1.7 & 1.0 & 1.0 & 1.0 & 0.9 & 1.2 & 0.9 & 1.0 & 1.0 & 1.0 & 10.8 \\
\hline & & 1 & 1.0 & 1.1 & 1.0 & 1.0 & 1.0 & 1.0 & 0.8 & 1.0 & 0.8 & 0.8 & 9.4 \\
\hline & & 2 & 1.0 & 1.1 & 1.2 & 1.0 & 1.0 & 0.9 & 1.0 & 0.9 & 1.0 & 1.0 & 10.2 \\
\hline & & 3 & 1.0 & 0.9 & 0.9 & 1.0 & 1.1 & 0.9 & 1.0 & 1.0 & 1.0 & 0.9 & 9.7 \\
\hline & & 4 & 1.0 & 0.9 & 1.0 & 1.0 & 1.3 & 1.0 & 1.0 & 0.9 & 1.2 & 1.0 & 10.4 \\
\hline & & 5 & 1.1 & 0.9 & 1.0 & 0.9 & 1.0 & 1.3 & 0.9 & 1.0 & 1.1 & 1.0 & 10.1 \\
\hline & & 6 & 0.9 & 0.8 & 1.0 & 1.0 & 1.0 & 0.9 & 1.0 & 0.9 & 0.9 & 0.9 & 9.3 \\
\hline & & 7 & 0.9 & 0.9 & 0.9 & 1.0 & 0.9 & 1.0 & 1.1 & 1.1 & 0.9 & 1.0 & 9.7 \\
\hline & & 8 & 1.1 & 1.1 & 1.0 & 1.1 & 1.0 & 1.0 & 1.0 & 1.0 & 1.3 & 1.0 & 10.5 \\
\hline & & 9 & 1.0 & 0.9 & 1.0 & 1.0 & 1.0 & 1.0 & 1.0 & 0.9 & 0.9 & 1.2 & 9.9 \\
\hline & Total & & 10.7 & 9.5 & 10.1 & 10.0 & 10.2 & 10.2 & 9.6 & 9.8 & 10.2 & 9.7 & 100.0 \\
\hline
\end{tabular}

BP $(10.7 \%)$ compared with $1960(9.5 \%)$ in the automatic-transmission dataset. Similarly, for diastolic BP, 4744 zero end digits $(10.7 \%)$ were observed in the patient-texted dataset compared with 1977 (9.5\%) in the automatic-transmission dataset.

Table 2 shows a cross tabulation of the percentages of systolic and diastolic end digits for the patient-texted and automatic-transmission datasets, with percentages calculated out of the total number of $\mathrm{BP}$ readings.

Table 2 shows that $1.7 \%$ of the BP readings had a double-zero end digit, whereas the majority of the other end-digit combinations were in the range of
$0.9 \%-1.2 \%$. If the end digits were entirely random, then we would expect a percentage of about $1 \%$ in each cell, and unsurprisingly the automatic-transmission system is consistent with this supposition. Out of all patients with a diastolic reading ending in $0,16 \%$ also had a systolic reading ending in 0 (and vice versa).

\section{GEE analysis}

The results from the GEE analysis are shown in table 3.

The odds of double zeros in the patient-texted population were approximately twice those in the automatic-transmission (OR 2.1, 95\% CI 1.7 to 2.6). Our conclusions

Table 3 ORs for the comparison of patient-texted versus automatic-transmission from a generalised estimating equations model fitted to the repeated measures outcome data

\begin{tabular}{llclcc}
\hline Outcome & Automatic-transmission & Patient-texted & OR & 95\% Cl of OR & Robust Z scores \\
\hline Double zero & $0.9 \%(177 / 20705)$ & $1.7 \%(761 / 44150)$ & 2.12 & 1.72 to 2.62 & 7.07 \\
Systolic end digit of zero & $9.5 \%(1960 / 20705)$ & $10.7 \%(4727 / 44150)$ & 1.15 & 1.08 to 1.22 & 4.53 \\
Diastolic end digit of zero & $9.5 \%(1977 / 20705)$ & $10.7 \%(4744 / 44150)$ & 1.17 & 1.10 to 1.25 & 4.72 \\
Systolic 134* & $4.1 \%(266 / 6478)$ & $6.3 \%(979 / 15484)$ & 1.52 & 1.28 to 1.82 & 4.70 \\
Diastolic 84† & $5.5 \%(299 / 5450)$ & $8.8 \%(795 / 9083)$ & 1.54 & 1.28 to 1.86 & 4.48 \\
\hline
\end{tabular}

*Only includes patients using the standard protocol with median systolic blood pressure in the range of 130-138.

†Only includes patients using the standard protocol with median diastolic blood pressure in the range of 82- 86. 
were unchanged when restricting the sample to highly engaged patients recording over 50 readings (OR 1.8, $95 \%$ CI 1.5 to 2.1 , see online supplementary file 1 ), or when restricting both samples to have median BP above $120 / 80$ (OR 1.9 , 95\% CI 1.6 to 2.4 , see online supplementary file 2$)$.

We also found evidence of specific-value preference for systolic 134 and diastolic 84 in patients following the standard protocol (see table 3), presumably due to the threshold for receiving alerts of 135/85 in the patient-texted system. Again, this conclusion remained unchanged after considering highly engaged patients recording 50 readings or above (see online supplementary file 1).

\section{DISCUSSION}

We found evidence of end-digit preference for 'zero' $\mathrm{BP}$ readings in the patient-texted population when compared with the automatic-transmission cohort. Zero end digits tended to occur together, suggesting that end-digit preference is often expressed for both diastolic and systolic readings simultaneously. This is consistent with the literature which shows strong evidence of zero end-digit preference among primary care professionals taking BP measurements. For example, Lyratzopoulos $e t a l^{22}$ examined the quality of measurements in routinely collected data, and did not find any evidence of digit preference for the final digits of height, weight or cholesterol measurements taken by health professionals, but did find a strong zero end-digit preference for systolic and diastolic BP measurements.

We also found evidence of specific-value preference below the hypertension threshold (in non-diabetics) of $135 / 85$. This finding differs from some studies ${ }^{17} 24$ which did not find specific-value preference near treatment thresholds. This may be because in our study, patients using the patient-texted system received automated messages when their readings breached the threshold. Specific-value selection of readings below the threshold may not always be due to inaccuracy or inappropriate rounding; anecdotally, some patients faced with a higher than expected BP will repeat the measurement until they achieve an acceptable reading.

Our results were unchanged after a sensitivity analysis restricting the data to those patients recording more than 50 readings. This suggests that high engagement and familiarity with a system does not eliminate end-digit preference or specific-value preference, and that value preference remains even when restricting the sample to those who are most enthusiastic and highly engaged with the study. This finding is consistent with the results from the EffetS d'une intervention multifaCtorielle sur les fActeurs de risque des patients hyPErtendus(ESCAPE) cluster randomised controlled trial ${ }^{18}$ which suggest that end-digit preference does not diminish, and may actually increase over time as patients become more familiar with the use of electronic devices, and aware of thresholds. ${ }^{18}$

Nevertheless, despite the statistically significant results, they are unlikely to be clinically relevant: in the patient-texted group, the overall percentage of doublezero readings was still below $2 \%$, and occurrences of specific-value selection of systolic 134 and diastolic 84 were still within $4 \%$ of the automated system. However, it has been suggested that even very small differences could affect patient care. ${ }^{16} 1923$ Hence, it may be important to inform patients before using the telemonitoring system that accuracy of readings is important, and then the onus will be on the patient to provide accurate readings to ensure appropriate care.

A strength of our study is the very large sample size of almost 65000 BP readings across 1267 patients in total; therefore, we were well powered to detect potential end-digit preference. Patients were recruited from routine practice so should be representative of the patients who would participate in a telemonitoring service, although recruitment only took place in Scotland and so the results may not be generalisable to patients in other countries. Another advantage of our study was that we were able to compare with individual level data from a cohort of patients taking their readings at home but with no possibility of data manipulation. Most studies in the literature do not have a suitable comparison group and therefore have to rely on methods to detect end-digit preference within single samples. However, because of differences in the eligibility criteria, our patient populations had significantly different levels of BP control making comparison challenging, especially for the analysis of specific-value preference. In particular, patients with CKD (stages 3-5) or who had diabetes were included in the patient-texted cohort but were excluded from the automatic-transmission cohort. This made it difficult to establish specific-value preference below the BP thresholds since any such apparent 'preference' may be confounded with the observed differences between populations. However, our GEE analysis attempted to address this by confining the samples in both groups to only include patients with median BPs within a restricted range and using a standard protocol (ie, excluding patients with CKD and diabetes). For the end-digit preference analysis more generally, in sensitivity analysis we restricted the samples to patients with median BP above 120/80 and our conclusions were unchanged.

In conclusion, our results provide evidence for the existence of zero end-digit preference and specific-value preference among patients self-reporting their BP using telemonitoring. However, the proportion of readings affected by this is small and unlikely to be clinically important. The extent of end-digit preference is not so great as to warrant the use of more expensive systems that allow the automatic transfer of BP readings, and so telemonitoring services can continue to use manual entry systems. However, we recommend that 
patients are reminded to record readings as accurately as possible to achieve appropriate care.

Acknowledgements We would like to thank Mohammad Al-remal for his preliminary analysis of an earlier dataset, which helped us to design and plan for the analysis reported in this paper. RAP is supported in this work by NHS Lothian via the Edinburgh Clinical Trials Unit.

Contributors BM, PP, HP, JH and AS made substantial contributions to the conception and design of the work. MP and VSH monitored the implementation of the telemonitoring system and co-ordinated the study. MP acquired the raw data. RAP planned and performed the statistical analysis. RAP wrote the first draft of the paper. All authors revised and commented on the manuscript.

Funding This work was supported by the Chief Scientists Office of the Scottish Government (reference number CZH/4/1135). The Scale-Up BP project is supported by the Scottish Government Technology Enabled Care Programme and the HITS study was supported by the BUPA foundation (reference number 748/G24).

Competing interests None declared.

Patient consent Obtained.

Ethics approval The HITS trial had ethical approval from the South East Scotland Research Ethics Committee (reference number 08/S1101/38).

Provenance and peer review Not commissioned; externally peer reviewed.

Data sharing statement The dataset used in this study is available for download from figshare, D0l: 10.6084/m9.figshare. 5363428

Open Access This is an Open Access article distributed in accordance with the Creative Commons Attribution Non Commercial (CC BY-NC 4.0) license, which permits others to distribute, remix, adapt, build upon this work non-commercially, and license their derivative works on different terms, provided the original work is properly cited and the use is non-commercial. See: http://creativecommons.org/ licenses/by-nc/4.0/

(c) Article author(s) (or their employer(s) unless otherwise stated in the text of the article) 2018. All rights reserved. No commercial use is permitted unless otherwise expressly granted.

\section{REFERENCES}

1. Lawes CM, Vander Hoorn S, Rodgers A, et al. Global burden of blood-pressure-related disease, 2001. Lancet 2008;371:1513-8.

2. Pickering TG, Miller NH, Ogedegbe G, et al. Call to action on use and reimbursement for home blood pressure monitoring: a joint scientific statement from the American Heart Association, American Society Of Hypertension, and Preventive Cardiovascular Nurses Association. Hypertension 2008;52:10-29.

3. Serumaga B, Ross-Degnan D, Avery AJ, et al. Effect of pay for performance on the management and outcomes of hypertension in the United Kingdom: interrupted time series study. BMJ 2011;342:d108.

4. Okonofua EC, Simpson KN, Jesri A, et al. Therapeutic inertia is an impediment to achieving the healthy people 2010 blood pressure control goals. Hypertension 2006;47:345-51.

5. Osterberg L, Blaschke T. Adherence to medication. N Engl J Med 2005;353:487-97.

6. Keenan K, Hayen A, Neal BC, et al. Long term monitoring in patients receiving treatment to lower blood pressure: analysis of data from placebo controlled randomised controlled trial. BMJ 2009;338:b1492.

7. Stergiou GS, Baibas NM, Gantzarou AP, et al. Reproducibility of home, ambulatory, and clinic blood pressure: implications for the design of trials for the assessment of antihypertensive drug efficacy. Am J Hypertens 2002;15:101-4.

8. Dolan E, Stanton A, Thijs L, et al. Superiority of ambulatory over clinic blood pressure measurement in predicting mortality: the Dublin outcome study. Hypertension 2005;46:156-61.

9. Pickering TG, Hall JE, Appel LJ, et al. Recommendations for blood pressure measurement in humans and experimental animals: part 1: blood pressure measurement in humans: a statement for professionals from the Subcommittee of Professional and Public Education of the American Heart Association Council on High Blood Pressure Research. Hypertension 2005;45:142-61.
10. Ringrose J, Padwal R. The ongoing saga of poor blood pressure measurement: past, present, and future perspectives. J Clin Hypertens 2017;19:611-3.

11. Omboni S, Gazzola T, Carabelli G, et al. Clinical usefulness and cost effectiveness of home blood pressure telemonitoring: meta-analysis of randomized controlled studies. J Hypertens 2013;31:455-67.

12. Tucker KL, Sheppard JP, Stevens R, et al. Self-monitoring of blood pressure in hypertension: A systematic review and individual patient data meta-analysis. PLoS Med 2017;14:e1002389.

13. McKinstry B, Hanley J, Wild S, et al. Telemonitoring based service redesign for the management of uncontrolled hypertension: multicentre randomised controlled trial. BMJ 2013;346:f3030.

14. Alsanjari ON, de Lusignan S, van Vlymen J, et al. Trends and transient change in end-digit preference in blood pressure recording: studies of sequential and longitudinal collected primary care data. Int $J$ Clin Pract 2012;66:37-43.

15. Ayodele OE, Sanya EO, Okunola OO, et al. End digit preference in blood pressure measurement in a hypertension specialty clinic in southwest Nigeria. Cardiovasc J Afr 2012;23:85-9.

16. Broad J, Wells S, Marshall R, et al. Zero end-digit preference in recorded blood pressure and its impact on classification of patients for pharmacologic management in primary care - PREDICT-CVD-6. Br J Gen Pract 2007;57:897-903.

17. de Lusignan S, Belsey J, Hague N, et al. End-digit preference in blood pressure recordings of patients with ischaemic heart disease in primary care. J Hum Hypertens 2004;18:261-5.

18. Dibao-Dina C, Lebeau JP, Huas D, et al. ESCAPE ancillary blood pressure measurement study 2: changes in end-digit preference after 2 years of a cluster randomized trial. Blood Press Monit 2015;20:346-50.

19. Harrison WN, Lancashire RJ, Marshall TP. Variation in recorded blood pressure terminal digit bias in general practice. J Hum Hypertens 2008:22:163-7.

20. Jie G, Jian W, Qiaowen $\mathrm{H}$, et al. Investigation of end-digit preference in blood pressure records of hospitalized chinese patients and analysis of risk factors. Postgrad Med 2012;124:53-7.

21. Kim ES, Samuels TA, Yeh HC, et al. End-digit preference and the quality of blood pressure monitoring in diabetic adults. Diabetes Care 2007;30:1959-63.

22. Lyratzopoulos G, Heller RF, Hanily M, et al. Risk factor measurement quality in primary care routine data was variable but nondifferential between individuals. J Clin Epidemiol 2008;61:261-7.

23. Nietert PJ, Wessell AM, Feifer C, et al. Effect of terminal digit preference on blood pressure measurement and treatment in primary care. Am J Hypertens 2006;19:147-52.

24. Wang $Y$, Wang $Y$, Qain $Y$, et al. Longitudinal change in enddigit preference in blood pressure recordings of patients with hypertension in primary care clinics: Minhang study. Blood Press Monit 2015;20:74-8.

25. Wen SW, Kramer MS, Hoey J, et al. Terminal digit preference, random error, and bias in routine clinical measurement of blood pressure. J Clin Epidemiol 1993;46:1187-93.

26. Kallioinen N, Hill A, Horswill MS, et al. Sources of inaccuracy in the measurement of adult patients' resting blood pressure in clinical settings: a systematic review. J Hypertens 2017;35:421-41.

27. Chatellier G, Dutrey-Dupagne C, Vaur L, et al. Home self blood pressure measurement in general practice. The SMART study. Selfmeasurement for the Assessment of the Response to Trandolapril. Am J Hypertens 1996;9:644-52.

28. Steventon A, Chaudhry SI, Lin Z, et al. Assessing the reliability of self-reported weight for the management of heart failure: application of fraud detection methods to a randomised trial of telemonitoring. BMC Med Inform Decis Mak 2017;17:43.

29. Omron Healthcare website. https://www.omron-healthcare.com/eng p/products/bloodpressuremonitoring (accessed 22 Aug 2017).

30. Westhoff TH, Schmidt S, Zidek W, et al. Validation of the Stabil-OGraph blood pressure self-measurement device. J Hum Hypertens 2008;22:233-5.

31. Carey VJ. Ported to R by Thomas Lumley and Brian Ripley. gee: generalized estimation equation solver. R Package version 4.13-19. 2015 https://CRAN.R-project.org/package=gee.

32. R Core Team. R: A language and environment for statistical computing. Vienna, Austria: R Foundation for Statistical Computing, 2016.

33. IBM Corp. Released 2012. IBM SPSS statistics for windows, version 21.0. Armonk, NY: IBM Corp, 2012. 